\title{
Numerical test of the Gribov-Zwanziger scenario in Landau gauge
}

\author{
Attilio Cucchieri \\ Instituto de Física de São Carlos, Universidade de São Paulo, \\ Caixa Postal 369, 13560-970 São Carlos, SP, Brazil \\ E-mail: attilio@ifsc.usp.br

\section{Tereza Mendes*} \\ Instituto de Física de São Carlos, Universidade de São Paulo, \\ Caixa Postal 369, 13560-970 São Carlos, SP, Brazil \\ E-mail: mendes@ifsc.usp.br
}

We review the status of lattice simulations of gluon and ghost propagators in Landau gauge, testing predictions of the Gribov-Zwanziger confinement scenario.

International Workshop on QCD Green's Functions, Confinement, and Phenomenology - QCD-TNT09 September 07 - 112009

ECT Trento, Italy

*Speaker. 


\section{Introduction}

The investigation of infrared properties of Landau-gauge gluon and ghost propagators - in order to test predictions of the Gribov-Zwanziger confinement scenario, or of the Kugo-Ojima scenario - has generated a flurry of papers in the last couple of years. While the formulation of these two scenarios, their equivalence (or lack thereof) and the chosen procedure for solving these propagators' Dyson-Schwinger equations have become matters of heated debate, a consistent picture emerges from lattice studies. Based on this picture, which is in partial disagreement with the predictions of both scenarios, present activity focuses on critical revisions of the original scenarios, on discussion of their main assumptions and/or implications, and on whether or not there are physical criteria to prefer a solution of the Dyson-Schwinger equations (or other functional methods) of the "scaling" or of the "massive" type. In this way, one hopes to have gained a deeper insight into confinement in Landau gauge. We will not review these various analytic studies here, but rather refer to recent status reports and overviews contained in these proceedings, such as Ref. [1], which summarizes the so-called refined Gribov-Zwanziger framework, Refs. [2] and [3], which address the problem of the characterization of the Kugo-Ojima scenario and its relation to the Gribov-Zwanziger one, or Ref. [4], which reviews the solution of Dyson-Schwinger equations in Landau gauge and discusses phenomenological applications. We do, however, attempt to review thoroughly the recent literature on lattice studies of the topic.

In what follows we consider tests of the original Gribov-Zwanziger confinement scenario, i.e. a vanishing gluon propagator and an enhanced ghost propagator in the infrared limit. On the contrary, in the refined Gribov-Zwanziger framework mentioned above, one expects a finite (nonzero) gluon propagator and a free ghost propagator in the same limit. These two cases are consistent respectively with the so-called scaling and massive (or decoupling) solutions of the Dyson-Schwinger equations. The latter behavior has been strongly favored in all recent extensive lattice studies, performed on very large lattices for pure SU(2) and SU(3) gauge theory. As argued in [5], the essential features of the original Gribov-Zwanziger confinement scenario are not incompatible with these findings, since violation of reflection positivity is clearly observed for the gluon propagator and enhancement with respect to the free propagator is seen for the ghost at intermediate momenta. Also, a logarithmic enhancement of the ghost propagator might be possible in the continuum limit. A scaling solution (with nontrivial infrared exponents), however, is ruled out.

From the point of view of lattice simulations, we must strive to keep under control the various sources of systematic errors that might obscure the true infrared behavior of the propagators, in order to conclude that the behavior described above is firmly established. Of course, once the massive behavior is confirmed from the simulations, one must understand why this behavior arises in the infrared limit of the theory, and how we might reconcile it with a confinement mechanism. Again, insight into the problem can hopefully come from the numerical simulations themselves. Also, it should be noted that the comparisons mentioned above assume that the gauge definition on the lattice is physically equivalent to the continuum one, an issue that should be carefully investigated. We will refer here to the so-called minimal Landau gauge condition [6].

In Section 2 we review some aspects of the numerical simulations, with special attention to the main possible sources of systematic errors. We also attempt an overview of references and chronology of the main recent lattice results on the topic. In Section 3 we summarize interesting constraints 
on the infrared behavior of the propagators, written in the form of upper and lower bounds at fixed lattice volume. Although these bounds were introduced as a guide to the infinite-volume extrapolation, we believe they will be useful tools to investigate why the propagators have the observed behavior, since they naturally relate to a statistical interpretation of the gluon propagator and to a clearer view of the ghost propagator in terms of the spectrum of the Faddeev-Popov operator. Section 4 is dedicated to the results from very large lattices mentioned above. We summarize the analysis of data from our simulations of the pure-SU(2) case, which are essentially equivalent to the corresponding results by other lattice groups. In Section 5 we address the $\beta=0$ case, where various sources of systematic errors may be investigated more easily. The observed behavior is compared with the one at finite $\beta$. Finally, we present our conclusions in Section 6.

\section{The simulations}

On the lattice, the gauge action is written in terms of oriented plaquettes, formed by the link variables $U_{\mu}(x)$, which are elements of the gauge group $S U\left(N_{c}\right)$ and change under gauge transformations as

$$
U_{\mu}(x) \rightarrow U_{\mu}^{g}(x) \equiv g(x) U_{\mu}(x) g(x+\hat{\mu})^{\dagger},
$$

where $g \in S U\left(N_{c}\right)$, lattice sites are labeled by $x$ and $\mu$ refers to the directions along the lattice. Consequently, all closed loops are gauge-invariant quantities, including the plaquettes in the puregauge-theory action

$$
S(\{U\})=\frac{\beta}{N_{c}} \sum_{\mu<v} \sum_{x} \Re \operatorname{Tr}\left[11-U_{\mu}(x) U_{v}(x+\hat{\mu}) U_{\mu}^{\dagger}(x+\hat{v}) U_{v}^{\dagger}(x)\right] .
$$

Here $\beta$ is the lattice parameter, related to the bare coupling constant $g_{0}^{2}$ as $\beta=2 N_{c} / g_{0}^{2}$. Except where otherwise indicated, we consider (symmetric) hypercubic lattices of four space-time dimensions. The lattice size in physical units is given by $L=N a$, where $N$ is the number of points per lattice direction and the lattice spacing $a$ is expressed in physical units. The physical volume is thus $V=L^{4}$. (Note that one often refers to the "lattice volume" $N^{4}=V / a^{4}$.) We assume periodic boundary conditions.

Let us remark that the resulting path integral has a finite (group) integration volume and there is in principle no need for gauge fixing on the lattice. A procedure for fixing the gauge numerically may nevertheless be implemented in a straightforward way in the simulation, without the need to consider the Faddeev-Popov matrix $\mathscr{M}$. For minimal Landau gauge, one minimizes the functional

$$
\mathscr{E}(\{g\})=1-\frac{a^{4}}{4 N_{c} V} \sum_{\mu} \sum_{x} \Re \operatorname{Tr} U_{\mu}^{g}(x)
$$

with respect to the gauge transformations $\{g\}$. Indeed, the first derivative of this functional yields the familiar Landau gauge condition of null (lattice) divergence of the gauge field, which is defined in terms of the link variables as

$$
A_{\mu}(x)=\frac{1}{2 i a g_{0}}\left[U_{\mu}(x)-U_{\mu}^{\dagger}(x)\right]_{\text {traceless }} .
$$


The prescription for fixing the gauge in a simulation is thus to: 1) produce a gauge-link configuration $\{U\}$ as usual, 2) to find $\{g\}$ that is a (local) minimum of the functional $\mathscr{E}$ (holding $\{U\}$ fixed) and 3) to transform $\{U\}$ following Eq. (2.1) for the selected $\{g\}$. The resulting configuration is a gauge-fixed realization of the link variables, which will be used to compute observables of interest such as the gluon and ghost propagators.

The gluon propagator is given in Landau gauge simply by

$$
D_{\mu v}^{a b}(p)=\sum_{x} e^{-2 i \pi p \cdot x}\left\langle A_{\mu}^{a}(x) A_{v}^{b}(0)\right\rangle=\delta^{a b}\left(g_{\mu v}-\frac{p_{\mu} p_{v}}{p^{2}}\right) D\left(p^{2}\right),
$$

where $p$ is the momentum and $a, b$ are color indices. It is therefore determined solely by the scalar function $D\left(q^{2}\right)$ associated to its transverse component. In the original Gribov-Zwanziger scenario, gluon confinement is associated with violation of reflection positivity for the gluon propagator (in real space-time).

As pointed out above, lattice gauge fixing is accomplished without the need to compute the Faddeev-Popov matrix $\mathscr{M}$. Nevertheless, the matrix can be obtained directly from the second variation of the gauge-fixing functional $\mathscr{E}$, which corresponds to the Jacobian of the gauge-fixing condition. It is interesting to note that in this way there is also no need to consider the ghost fields explicitly [7]. The ghost propagator $G\left(p^{2}\right)$ is given by the inverse of $\mathscr{M}$ as

$$
G\left(p^{2}\right)=\frac{1}{N_{c}^{2}-1} \sum_{x, y, a} \frac{e^{-2 \pi i p \cdot(x-y)}}{V}\left\langle\mathscr{M}^{-1}(a, x ; a, y)\right\rangle .
$$

An infrared enhancement of $G\left(p^{2}\right)$ with respect to the tree-level ghost propagator $G\left(p^{2}\right) \sim p^{-2}$ is expected in the original Gribov-Zwanziger scenario (and in the Kugo-Ojima one) as a sign of confinement.

Let us note that the known problem of Gribov copies is present on the lattice as well, since each local minimum of the functional $\mathscr{E}$ corresponds to an equivalent (lattice) gauge copy. The algorithm for fixing $\{g\}$ has in principle no control over which copy gets selected. Because of the minimization, we know that $\mathscr{M}$ is positive semi-definite and, as a result, the sampled copies are inside the first Gribov horizon $\Omega$, which is delimited by the vanishing of $\lambda_{\min }$, the smallest nontrivial eigenvalue of $\mathscr{M}$. It is usually argued that a unique copy might be obtained, corresponding to the fundamental modular region $\Lambda$, if one were able to determine the global minimum of $\mathscr{E}$. This region has been studied on the lattice in [8]. In any case, $\Omega$ and $\Lambda$ are shown to be convex regions of very high dimensionality, which likely constrains the statistical weight of gauge configurations to lie near their boundary. In particular, one should check if the sampled configurations have vanishing $\lambda_{\min }$ as the lattice volume goes to infinity.

Lattice simulations have been carried out since the mid 1980s for the gluon propagator [9] and since the mid 1990s for the ghost [10] (see also [11]). Early studies have established that the gluon propagator is not enhanced at small momenta [12], but did not allow further conclusions about its infrared behavior. A turnover point in momentum (suggesting a null propagator) could only be seen at strong coupling [13] or in three space-time dimensions [14]. However, even in this simplified case, a later study on a very large lattice (of volume $140^{3}$ ) was still not conclusive [15], although it was possible to fit $D(0)$ to zero in an infinite-volume extrapolation, and violation of reflection positivity was clearly seen [16]. For the ghost propagator, infrared enhancement was 
observed, but the corresponding infrared exponent seemed to become smaller as lower momenta became available $[10,11,17,18]$. It was also clearly shown that $\lambda_{\min }$ goes to zero with increasing lattice volume $[19,18]$. These studies were complemented by investigations of the strong coupling constant (see e.g. [20, 21]) and several three-point vertices (see e.g. [22, 23, 24, 18]). This was the status until 2006. As became clear later, systematic effects had not yet been properly taken into account, which limited the conclusions (or the lack thereof) of these studies. We now pause for a moment and list the main such possible effects (and related references) below.

- Gribov-copy effects: this is a very important issue. As commented above, usual simulations do not take fluctuations in the values of the propagators due to Gribov-copy effects into account. A few studies have considered the determination of the absolute minimum of the gauge-fixing functional (see e.g. [11, 25]) or other criteria to fix the gauge [26]. It was generally found that the effect of Gribov copies decreases as the lattice volume increases. This statement must be taken with a grain of salt, since the number of copies surveyed is limited and one does not know for sure if this number is large enough at a given volume to allow the determination of the global minimum. Studies of the exact structure of Gribov copies are now being carried out (on small lattices) [27]. Let us mention that it was argued by Zwanziger [28] that averages taken in the fundamental modular region should coincide with averages in $\Omega$ in the infinite-volume limit. We thus conclude that a sign of significant Gribovcopy effects has not yet been seen and the effects observed so far are probably connected to the next item below. (We do note, however, that a very recent study has reported on sizeable effects at large lattice volumes [29].)

- Finite-volume effects: perhaps surprisingly, these are the most serious systematic effects we have to deal with. To be sure, lattice simulations must be carried out at finite lattice volumes, since computers have finite memory. As mentioned above, the physical extent of the lattice $L$ is given by the number of lattice points along each direction multiplied by the lattice spacing $a$ in physical units, which is directly related to the lattice parameter $\beta$. To simulate closer to the continuum limit one must go to smaller $a$, or equivalently to larger $\beta$, while keeping the simulated lattice large enough to represent the relevant energy scales of the problem. Strictly speaking, one would need an extrapolation to infinite lattice volume at each fixed value of $\beta$. (A continuum extrapolation would additionally require running at increasingly smaller values of $a$.) In usual lattice applications, though, taking the infinite-volume limit is not among the most serious issues, since one typically just needs to have a sufficient number of points to ensure a physical lattice size of the order of the relevant hadronic scale, i.e. around $1 \mathrm{fm}$. The main effort is then to go to very small $a$, in order to avoid discretization errors (addressed below). In studies of the infrared limit, however, the situation is different, and finite-size effects play an important role. This happens because the infrared limit lies at small $p$, corresponding to large $L$. (Note that the smallest nonzero momentum that can be represented on a lattice of side $L$ is $\sim 2 \pi / L$.)

- Discretization effects: as indicated above, the effects due to simulating at nonzero lattice spacing $a$ are not expected to be so serious in the infrared limit, because the energy scale associated with the cutoff $a$ (which is $\sim 1 / a$ ), is sufficiently high compared to the typical 
momenta of interest. In other words, the long wavelengths we are interested in do not resolve the lattice spacing and are not much affected by it. Nevertheless, discretization errors may be important for the breaking of rotational symmetry as well as for the possible different discretizations of the gluon field and of the gauge-fixing condition. There are ways to reduce effects due to the breaking of rotational symmetry, such as cutting out the momenta characterized by large effects [12] (the so-called cylindrical cut), improving the lattice definition of the momenta [30] and including (hypercubic) corrections into the momentum-dependence of the Green's functions [31]. As for the discretization of the gluon field and of the lattice Landau gauge condition, several different definitions may be considered (see e.g. [32]). These studies have usually found that different discretization procedures lead to gluon propagators that differ only by a multiplicative constant, which can be reabsorbed in the (multiplicative) renormalization of the propagator.

- Unquenching: in the Gribov-Zwanziger and related scenarios, one hopes to get an understanding of confinement in the static-quark limit, where there is no string breaking and the confinement problem may be phrased as a search for explaining why an area law develops [33]. Thus, it should be sufficient to consider the pure-gauge theory treated here, also known as the quenched approximation. Nevertheless, an important question is how the picture gets affected once dynamical quarks are introduced in the simulations. Studies done so far (on relatively small lattices) show qualitatively the same behavior as in the pure-gauge case [34].

In 2007, studies of Dyson-Schwinger equations on the torus [35] hinted that finite-size effects might indeed be plaguing results from lattice simulations and predicted that physical lattice sides of the order of $15 \mathrm{fm}$ might be needed to begin to see the expected (conformal scaling) infrared behavior of the propagators. At about the same time, two other predictions of similar studies were verified in simulations: the (quantitative) equivalence of infrared propagators in the SU(2) and $\mathrm{SU}(3)$ cases $^{1}[37,38]$ and the verification of conformal scaling behavior in two space-time dimensions [39]. That same year, three groups came out with studies on very large lattices, which were all presented at the Lattice 2007 conference. The Berlin-Dubna group considered $80^{4}$ lattices, corresponding to a lattice extent of $13 \mathrm{fm}$, in the SU(3) case [40]. (Their study of the gluon propagator was later extended to $96^{4}$ lattices, corresponding to a lattice extent of $16 \mathrm{fm}$ [41].) The Adelaide group considered $112^{4}$ lattices, corresponding to $19 \mathrm{fm}$, in the SU(2) case [38]. Similarly, we considered $128^{4}$ lattices (corresponding to $27 \mathrm{fm}$ ) in the $\mathrm{SU}(2)$ case, plus three-dimensional lattices of size $320^{3}$, corresponding to $85 \mathrm{fm}$ [42]. What these studies showed was puzzling. On the one hand, the large volumes clearly allowed a better view of the infrared picture; on the other, this view was nothing like what the authors had imagined it would be! In fact, going to large volumes in the hopes of seeing a null infrared gluon propagator not only established that $D(0)$ was not null (even in three dimensions), but also exposed the fact that the previously seen enhancement of the ghost propagator goes away at very small momenta, and the data are consistent with a flat ghost dressing function in the infrared limit.

\footnotetext{
${ }^{1}$ Although one cannot expect this to hold at high values of $p$, it is conjectured that the cases $N_{c}=2$ and $N_{c}=3$ have the same infrared behavior. A recent comparison along a wider range of momenta presented in [36] shows some discrepancies between the two cases.
} 
This behavior is analyzed in Section 4. In the next section, on the other hand, we comment on rigorous bounds for the propagators, introduced as a guide to the infinite-volume extrapolation.

\section{Bounds on propagators and statistical interpretation}

As discussed in the previous section, one of the main difficulties in the lattice simulations is the extrapolation of gluon- and ghost-propagator data to infinite lattice volume. In fact, the correct volume dependence of the data may not be easily inferred from the behavior on medium-size (or even very large) lattices, especially since some quantities, such as the zero-momentum gluon propagator, are quite noisy. It is then very helpful to obtain constraints on the infrared behavior of the propagators, as the upper and lower bounds discussed in this section. We remark that these bounds are valid at each lattice volume and must be extrapolated to infinite volume, just as for the propagators. The advantage is that the bounds are written in terms of quantities that are more intuitive than the propagators themselves, making it easier to guess the expected volume dependence of the propagators and possibly allowing an explanation of the infrared behavior observed in the data.

In the case of the gluon propagator, we obtain [43] the bounds

$$
V\langle M(0)\rangle^{2} \leq D(0) \leq V d\left(N_{c}^{2}-1\right)\left\langle M(0)^{2}\right\rangle,
$$

where $d$ is the dimension, $V$ is the volume,

$$
D(0)=\frac{V}{d\left(N_{c}^{2}-1\right)} \sum_{\mu, b}\left\langle\left|\widetilde{A}_{\mu}^{b}(0)\right|^{2}\right\rangle
$$

is the zero-momentum propagator and $M(0)$ is defined as

$$
M(0)=\frac{1}{d\left(N_{c}^{2}-1\right)} \sum_{b, \mu}\left|\widetilde{A}_{\mu}^{b}(0)\right| .
$$

Let us also define the "magnetization"

$$
M^{\prime}(0)=\frac{1}{d\left(N_{c}^{2}-1\right)} \sum_{b, \mu} \widetilde{A}_{\mu}^{b}(0)
$$

From the above definitions we can get a statistical interpretation for the quantity on the right-hand side of (3.1): it is essentially the susceptibility associated with the magnetization $M^{\prime}(0)$ (since the average of this magnetization vanishes, due to the residual global gauge symmetry). By analogy with a $d$-dimensional spin system one would thus expect to see $V\left\langle M(0)^{2}\right\rangle \sim$ const, i.e. the statistical variance of the magnetization is proportional to the inverse of the volume, a behavior known as selfaveraging. At the same time, considering the statistical fluctuations in the Monte Carlo sampling of $M(0)$, we would expect $\langle M(0)\rangle^{2}$ to have the same volume dependence as $\left\langle M(0)^{2}\right\rangle$ [43]. The simple statistical argument presented above suggests that both $\langle M(0)\rangle^{2}$ and $\left\langle M(0)^{2}\right\rangle$ should show a volume dependence as $1 / V$. On the other hand, this suppression with $1 / V$ is compensated by the volume factor for both bounds in Eq. (3.1). Consequently, if this suggested behavior for the susceptibilities is verified, $D(0)$ converges to a nonzero constant in the infinite-volume limit. As explained in the next section, this is what one observes in the simulations. Note that the bounds 
in Eq. (3.1) apply to any gauge and that they can be immediately extended to the case $D\left(p^{2}\right)$ with $p \neq 0$. We also note that a very interesting stochastic interpretation for the gluon propagator has been investigated in [44].

Also in the case of the ghost propagator, a more intuitive picture comes from noticing that in Landau gauge, for any nonzero momentum $p$, one finds [45]

$$
\frac{1}{N_{c}^{2}-1} \frac{1}{\lambda_{\min }} \sum_{a}\left|\widetilde{\psi}_{\min }(a, p)\right|^{2} \leq G\left(p^{2}\right) \leq \frac{1}{\lambda_{\min }},
$$

where $\lambda_{\min }$ is the smallest nonzero eigenvalue of the Faddeev-Popov operator $\mathscr{M}$ and $\widetilde{\psi}_{\min }(a, p)$ is the corresponding eigenvector. Note that the upper bound is independent of the momentum $p$. If we now assume $\lambda_{\min } \sim L^{-v}$ and $G\left(p^{2}\right) \sim p^{-2-2 \kappa}$ at small $p$, we have that $2+2 \kappa \leq v$, i.e. $v>2$, is a necessary condition for the infrared enhancement of $G\left(p^{2}\right)$. A similar analysis can be carried out for a generic gauge condition. Consider the Gribov region $\Omega$, where all eigenvalues of $\mathscr{M}$ are positive. In the infinite-volume limit, as mentioned above, entropy favors configurations near the Gribov horizon $\partial \Omega$, where $\lambda_{\min }$ goes to zero. Thus, inequalities such as (3.5) can tell us if one should expect an enhanced $G\left(p^{2}\right)$ when the Boltzmann weight gets concentrated on $\partial \Omega$. (This answers the question posed in [46].) In other words, it is clearly necessary — but not sufficient to have a vanishing $\lambda_{\min }$ as the volume tends to infinity in order to observe enhancement of $G\left(p^{2}\right)$. The upper bound in Eq. (3.5) was tested for our data in [45], albeit with limited statistics for $\lambda_{\min }$. We find $v \approx 2$, consistent with finding $\kappa \rightarrow 0$ from fits of $G\left(p^{2}\right)$, as shown in the next section.

Let us also mention that a possible connection between the infrared behavior of the gluon propagator and the appearance of nontrivial zero modes of $\mathscr{M}$ for configurations near $\partial \Omega$ has been recently presented in [47]. The two types of scenarios obtained there can probably be related to the massive and the scaling solution for the propagators.

\section{Huge lattices}

Our study in the $S U$ (2) Landau case [45], using the very large lattices mentioned above, is summarized here. In Fig. 1 we show data for the gluon propagator in $d=4$ and $d=3$ for a wide range of (large) lattice volumes, indicating that $D(0)$ remains nonzero in the infinite-volume limit. Similar results are obtained in [38, 40, 41], as mentioned before, but also in [48], which takes Gribov-copy effects into account and in [49], which uses improved actions and anisotropic lattices. We have investigated the volume dependence of the bounds in Eq. (3.1) and found remarkably good agreement with the predicted $1 / V$ behavior for $\langle M(0)\rangle^{2}$ and $\left\langle M(0)^{2}\right\rangle$, thus implying a finite nonzero value for $D(0)$ in the infinite-volume limit. More precisely, by fitting the two quantities to $1 / V^{\alpha}$ we get the exponents $\alpha$ respectively $0.995(10)$ and $0.998(10)$. A similar analysis for the $\mathrm{SU}(3)$ case (considering somewhat smaller volumes) yields the exponents 1.058(6) and 1.056(6) [50]. Violation of reflection positivity for $D(x)$ is seen in all cases.

Our data for the ghost propagator support a tree-level (or free) form in the infrared limit. This behavior is better seen if one considers the dressing function $p^{2} G\left(p^{2}\right)$, as shown in Fig. 2. Indeed, the data can be well fitted [45] by the form $a-b\left[\log \left(1+c p^{2}\right)+d p^{2}\right] /\left(1+p^{2}\right)$, consistent with $\kappa=0$ in the infrared limit. Note also that, for smaller $p$, this form is equivalent to $a-b \log \left(1+c p^{2}\right)$ (proposed in [51]), where $c$ may be related to a gluon mass. This is also observed in $d=3$. We 

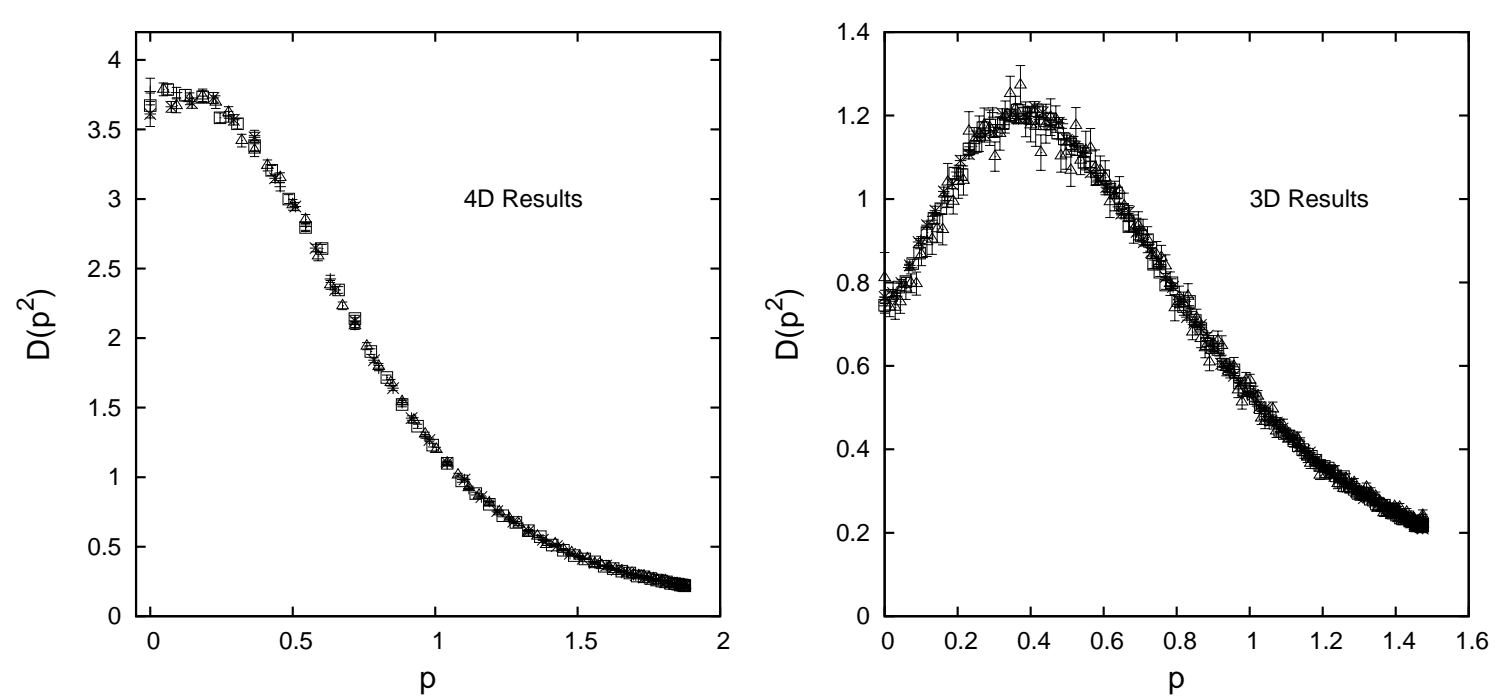

Figure 1: The gluon propagator $D\left(p^{2}\right)$ as a function of the lattice momenta $p$ (both in physical units) for the pure-SU(2) case in $d=4$ (left), considering volumes of up to $128^{4}$ (lattice extent $\sim 27 \mathrm{fm}$ ), and $d=3$ (right), considering volumes of up to $320^{3}$ (lattice extent $\sim 85 \mathrm{fm}$ ).

remark again that enhancement is seen at intermediate momenta and that, depending on how the fit parameters change with the lattice spacing, there might be a logarithmic enhancement in the continuum limit.

\section{5. $\beta=0$}

An interesting laboratory to test for various sources of systematic errors in the simulations is the apparently trivial case of $\beta=0$, i.e. no dynamics from the lattice Boltzmann weight associated with the action [see Eq. (2.1)]. This was considered in [13, 52, 53]. Since $\beta=0$ corresponds to an unphysical limit, the issue of setting the lattice scale (given by $a$ ) is delicate. As discussed in detail in [53], a possible choice is $a \rightarrow \infty$. This is convenient because the lattice extent will already be infinite and there will be no finite-size effects. On the other hand, if $a$ is large and $p$ is not very small there could be discretization effects. We must see from the data which effect is predominant. (The latter effect may also be measured in terms of breaking of rotational invariance.) As shown in [53], we see: 1) clear violation of reflection positivity for the gluon propagator, 2) a seemingly finite and nonzero limit for $D(0)$, including analysis with the bounds in Eq. (3.1), 3) a very good fit of $p^{2} G\left(p^{2}\right)$ to the form $a-b \log \left(1+c p^{2}\right)$ and 4) no finite-volume or rotational-symmetry-breaking effects, suggesting that the data are in the deep infrared limit and at infinite volume. Essentially, we see the same infrared behavior as for finite $\beta$. Note that our conclusions differ somewhat from [52].

\section{Conclusion}

Lattice simulations of infrared Landau gluon and ghost propagators have come a long way in the past couple of years. The current paradigm is that of a massive gluon and a free ghost, as 

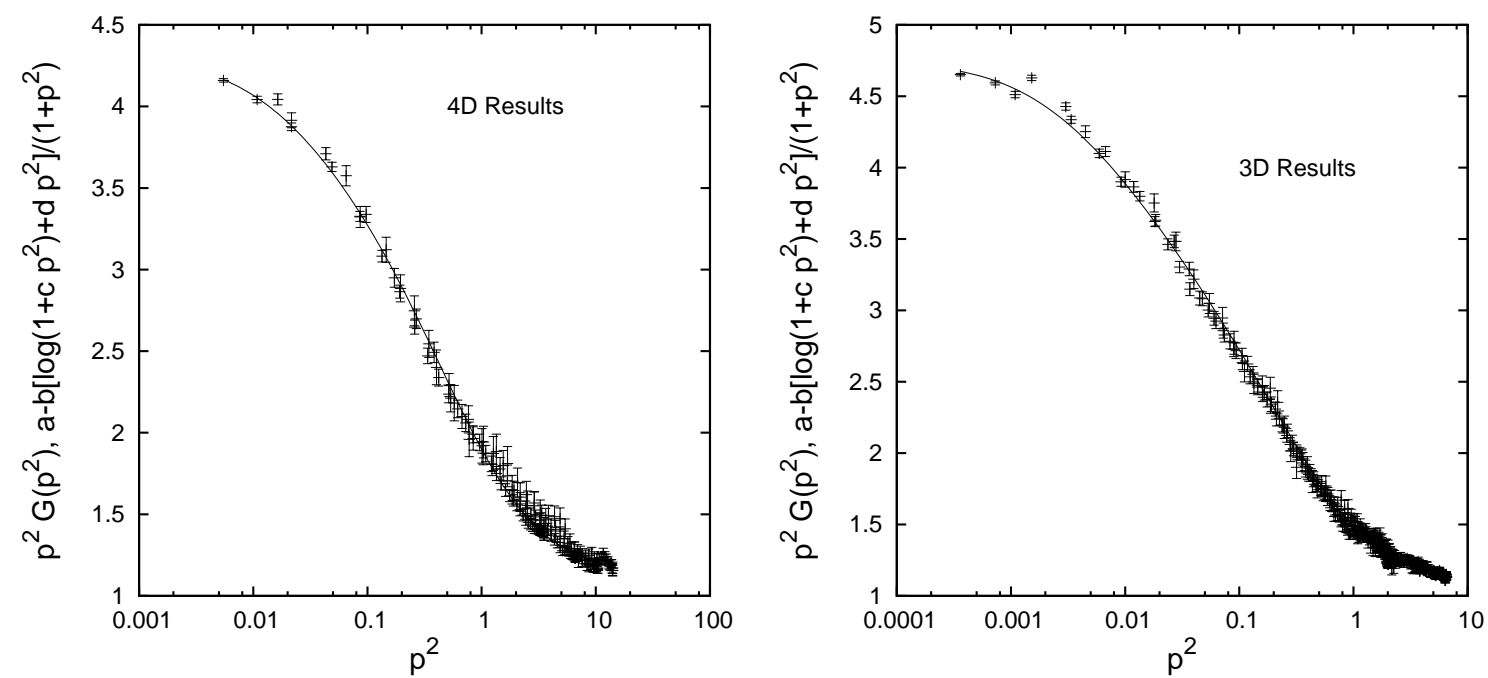

Figure 2: The ghost dressing function $p^{2} G\left(p^{2}\right)$ as a function of the lattice momenta $p^{2}$ (both in physical units) for the pure-SU(2) case in $d=4$ (left), considering volumes of up to $128^{4}$ (lattice extent $\sim 27 \mathrm{fm}$ ), and $d=3$ (right), considering volumes of up to $320^{3}$ (lattice extent $\sim 85 \mathrm{fm}$ ). Note the logarithmic scale for $p^{2}$. We also show the fits to $a-b\left[\log \left(1+c p^{2}\right)+d p^{2}\right] /\left(1+p^{2}\right)$. For $d=4$, the fit parameters $a, b, c, d$ are respectively $4.32(2), 0.38(1), 80(10), 8.2(3)$.

proposed long ago by some Dyson-Schwinger-equation studies (see e.g. [54]). These simulations are greatly influenced by interchange with researchers who use analytic and semi-analytic methods, and vice-versa. This synergy has contributed to making infrared QCD a very productive field, with an active community and several dedicated workshops every year.

Acknowledgements: The authors thank the organizers of QCD-TNT09 for a very stimulating meeting and acknowledge partial support from the Brazilian Funding Agencies FAPESP and CNPq. T.M. also thanks the Theory Group at DESY-Zeuthen for hospitality and the Alexander von Humboldt Foundation for financial support.

\section{References}

[1] D. Dudal, N. Vandersickel, H. Verschelde and S. P. Sorella, PoS QCD-TNT09, 012 (2009) [arXiv:0911.0082 [hep-th]].

[2] D. Binosi, PoS QCD-TNT09, 004 (2009) [arXiv:0911.0315 [hep-ph]].

[3] K. I. Kondo, PoS QCD-TNT09, 021 (2009) [arXiv:0911.2880 [hep-th]].

[4] A. A. Natale, PoS QCD-TNT09, 031 (2009) [arXiv:0910.5689 [hep-ph]].

[5] A. Cucchieri and T. Mendes, Proceedings of the XIII International Conference on Selected Problems of Modern Physics (Dubna, June 23-27, 2008), edited by B.M. Barbashov and S.M. Eliseev, JINR Dubna, 290 (2009) [arXiv:0809.2777 [hep-lat]].

[6] D. Zwanziger, Nucl. Phys. B 412, 657 (1994).

[7] J. E. Mandula, Phys. Rept. 315, 273 (1999) [arXiv:hep-lat/9907020]. 
[8] A. Cucchieri, Nucl. Phys. B 521, 365 (1998) [arXiv:hep-lat/9711024].

[9] J. E. Mandula and M. Ogilvie, Phys. Lett. B 185, 127 (1987).

[10] H. Suman and K. Schilling, Phys. Lett. B 373, 314 (1996) [arXiv:hep-lat/9512003].

[11] A. Cucchieri, Nucl. Phys. B 508, 353 (1997) [arXiv:hep-lat/9705005].

[12] D. B. Leinweber, J. I. Skullerud, A. G. Williams and C. Parrinello [UKQCD Collaboration], Phys. Rev. D 60, 094507 (1999) [Erratum-ibid. D 61, 079901 (2000)] [arXiv:hep-lat/9811027].

[13] A. Cucchieri, Phys. Lett. B 422, 233 (1998) [arXiv:hep-lat/9709015].

[14] A. Cucchieri, Phys. Rev. D 60, 034508 (1999) [arXiv:hep-lat/9902023].

[15] A. Cucchieri, T. Mendes and A. R. Taurines, Phys. Rev. D 67, 091502 (2003) [arXiv:hep-lat/0302022].

[16] A. Cucchieri, T. Mendes and A. R. Taurines, Phys. Rev. D 71, 051902 (2005) [arXiv:hep-lat/0406020].

[17] S. Furui and H. Nakajima, Phys. Rev. D 69, 074505 (2004) [arXiv:hep-lat/0305010]; J. Gattnar, K. Langfeld and H. Reinhardt, Phys. Rev. Lett. 93, 061601 (2004) [arXiv:hep-lat/0403011]; A. Sternbeck, E. M. Ilgenfritz, M. Mueller-Preussker and A. Schiller, Phys. Rev. D 72, 014507 (2005) [arXiv:hep-lat/0506007].

[18] A. Cucchieri, A. Maas and T. Mendes, Phys. Rev. D 74, 014503 (2006) [arXiv:hep-lat/0605011].

[19] A. Sternbeck, E. M. Ilgenfritz and M. Muller-Preussker, Phys. Rev. D 73, 014502 (2006) [arXiv:hep-lat/0510109].

[20] P. Boucaud et al., JHEP 9810, 017 (1998) [arXiv:hep-ph/9810322].

[21] J. C. R. Bloch, A. Cucchieri, K. Langfeld and T. Mendes, Nucl. Phys. B 687, 76 (2004) [arXiv:hep-lat/0312036].

[22] C. Parrinello, Phys. Rev. D 50, 4247 (1994) [arXiv:hep-lat/9405024].

[23] J. Skullerud and A. Kizilersu, JHEP 0209, 013 (2002) [arXiv:hep-ph/0205318].

[24] A. Cucchieri, T. Mendes and A. Mihara, JHEP 0412, 012 (2004) [arXiv:hep-lat/0408034].

[25] P. J. Silva and O. Oliveira, Nucl. Phys. B 690, 177 (2004) [arXiv:hep-lat/0403026]; A. Maas, Phys. Rev. D 79, 014505 (2009) [arXiv:0808.3047 [hep-lat]].

[26] I. L. Bogolubsky, G. Burgio, M. Muller-Preussker and V. K. Mitrjushkin, Phys. Rev. D 74, 034503 (2006) [arXiv:hep-lat/0511056]; A. Maas, arXiv:0907.5185 [hep-lat].

[27] D. Mehta, A. Sternbeck, L. von Smekal and A. G. Williams, PoS QCD-TNT09, 025 (2009) [arXiv:0912.0450 [hep-lat]].

[28] D. Zwanziger, Phys. Rev. D 69, 016002 (2004) [arXiv:hep-ph/0303028].

[29] I. L. Bogolubsky, E. M. Ilgenfritz, M. Muller-Preussker and A. Sternbeck, PoS LATTICE2009, 237 (2009) [arXiv:0912.2249 [hep-lat]].

[30] J. P. Ma, Mod. Phys. Lett. A 15, 229 (2000) [arXiv:hep-lat/9903009].

[31] F. de Soto and C. Roiesnel, JHEP 0709, 007 (2007) [arXiv:0705.3523 [hep-lat]]. 
[32] L. Giusti et al., Phys. Lett. B 432, 196 (1998) [arXiv:hep-lat/9803021]; F. D. R. Bonnet et al., Austral. J. Phys. 52, 939 (1999) [arXiv:hep-lat/9905006]; L. von Smekal, D. Mehta, A. Sternbeck and A. G. Williams, PoS LAT2007, 382 (2007) [arXiv:0710.2410 [hep-lat]].

[33] J. Greensite, Prog. Part. Nucl. Phys. 51, 1 (2003) [arXiv:hep-lat/0301023].

[34] P. Boucaud et al., Nucl. Phys. Proc. Suppl. 106, 266 (2002) [arXiv:hep-ph/0110171]; S. Furui and H. Nakajima, Few Body Syst. 40, 101 (2006) [arXiv:hep-lat/0503029]; E. M. Ilgenfritz et al., Braz. J. Phys. 37, 193 (2007) [arXiv:hep-lat/0609043]; P. O. Bowman et al., Phys. Rev. D 76, 094505 (2007) [arXiv:hep-lat/0703022].

[35] C. S. Fischer, A. Maas, J. M. Pawlowski and L. von Smekal, Annals Phys. 322, 2916 (2007) [arXiv:hep-ph/0701050].

[36] O. Oliveira and P. J. Silva, PoS QCD-TNT09, 033 (2009) arXiv:0911.1643 [hep-lat].

[37] A. Cucchieri, T. Mendes, O. Oliveira and P. J. Silva, Phys. Rev. D 76, 114507 (2007) [arXiv:0705.3367 [hep-lat]].

[38] A. Sternbeck, L. von Smekal, D. B. Leinweber and A. G. Williams, PoS LAT2007, 340 (2007) [arXiv:0710.1982 [hep-lat]].

[39] A. Maas, Phys. Rev. D 75, 116004 (2007) [arXiv:0704.0722 [hep-lat]].

[40] I. L. Bogolubsky, E. M. Ilgenfritz, M. Muller-Preussker and A. Sternbeck, PoS LAT2007, 290 (2007) [arXiv:0710.1968 [hep-lat]].

[41] I. L. Bogolubsky, E. M. Ilgenfritz, M. Muller-Preussker and A. Sternbeck, Phys. Lett. B 676, 69 (2009) [arXiv:0901.0736 [hep-lat]].

[42] A. Cucchieri and T. Mendes, PoS LAT2007, 297 (2007) [arXiv:0710.0412 [hep-lat]].

[43] A. Cucchieri and T. Mendes, Phys. Rev. Lett. 100, 241601 (2008) [arXiv:0712.3517 [hep-lat]].

[44] H. Suganuma, T. Iritani, A. Yamamoto and H. Iida, PoS QCD-TNT09, 044 (2009) arXiv:0912.0437 [hep-lat].

[45] A. Cucchieri and T. Mendes, arXiv:0804.2371 [hep-lat].

[46] A. Cucchieri, AIP Conf. Proc. 892, 22 (2007) [arXiv:hep-lat/0612004].

[47] J. Greensite, arXiv:1001.0784 [hep-th].

[48] V. G. Bornyakov, V. K. Mitrjushkin and M. Muller-Preussker, Phys. Rev. D 79, 074504 (2009) [arXiv:0812.2761 [hep-lat]].

[49] M. Gong, Y. Chen, G. Meng and C. Liu, Mod. Phys. Lett. A 24, 1925 (2009) [arXiv:0811.4635 [hep-lat]].

[50] O. Oliveira and P. J. Silva, Phys. Rev. D 79, 031501 (2009) [arXiv:0809.0258 [hep-lat]].

[51] A. C. Aguilar, D. Binosi and J. Papavassiliou, Phys. Rev. D 78, 025010 (2008) [arXiv:0802.1870 [hep-ph]].

[52] A. Sternbeck and L. von Smekal, arXiv:0811.4300 [hep-lat]; A. Maas et al., arXiv:0912.4203 [hep-lat].

[53] A. Cucchieri and T. Mendes, Phys. Rev. D 81, 016005 (2010) [arXiv:0904.4033 [hep-lat]].

[54] J. M. Cornwall, Phys. Rev. D 26, 1453 (1982); A. C. Aguilar and A. A. Natale, JHEP 0408, 057 (2004) [arXiv:hep-ph/0408254]; Ph. Boucaud et al., JHEP 0806, 099 (2008) [arXiv:0803.2161 [hep-ph]]. 